\title{
The photoreceptor sensory code for perceived duration during visual masking
}

\author{
GARY FELSTEIN and GERALD S. WASSERMAN \\ Sensory Coding Laboratory, Purdue University, West Lafayette, Indiana 47907
}

\begin{abstract}
In a study of the perceived duration of visually displayed letters that were either unmasked or masked by visual noise or homogeneous flashes, Haber and Standing (1970) found that the stimulus onset asynchrony limited the perceived duration of noise-masked letters presented in light adaptation but not of letters masked with homogeneous flashes in dark adaptation. Letters presented alone, as single flashes, exhibited adaptation-dependent visual persistence. The present psychobiological study replicated the stimulus conditions used by Haber and Standing while microelectrode recordings of sensory signals were taken from single Limulus photoreceptors. The data indicate that the sensory code-for perceived duration at the photoreceptor level is the time that the sensory signal is above a criterion potential. When adaptation is taken into account, this photoreceptor code replicates the complex functional relations found in the behavioral studies of Haber and Standing. The data suggest that neural integration causes an aftercoming mask to interrupt the sensory signal itself, not its processing, although processing may end when there is no signal left to be processed.
\end{abstract}

Haber and Standing (1970) reported that the perceived duration of a patterned target stimulus could be limited by the presentation of an aftercoming visual noise mask. They suggested that the mask might work through interruptive means by terminating the processing of the target stimulus representation. These suggestions were based on data from an experiment in which human observers were required to adjust the temporal positions of brief auditory clicks so that they coincided with the apparent onset and offset of the visual targets. This technique, first suggested by Sperling (1967), provides a measure of the perceived duration of a visual stimulus.

Using this technique, Haber and Standing measured the perceived durations of target stimuli whose objective durations varied between 10 and 1,000 msec. These measurements were made in dark- and light-adapted conditions and with or without aftercoming visual noise or homogeneous flash masks. The time between the onset of the target stimulus and that of the masking stimulus, or the stimulus onset asynchrony (SOA), was varied between 30 and $730 \mathrm{msec}$. Not every combination of these parameters was explored, however. In particular, the effect of noise masks was studied in a light-adapted state and the effect of homogeneous flash masks was studied in a dark-adapted state.

This work was supported by NSF Grant BNS 78-02359 and by a David Ross fellowship to Gary Felsten. Send reprint requests to Gerald S. Wasserman, Sensory Coding Laboratory, Department of Psychological Sciences, Purdue University, West Lafayette, Indiana 47907. Gary Felsten's present address is: Department of Anatomy, University of Illinois at the Medical Center, 1853 West Polk Street, Chicago, Illinois 60680.
Haber and Standing's results for the unmasked, dark-adapted condition indicated that the perceived duration was longer than the actual target duration for all target durations. The amount by which the perceived duration exceeds the stimulus duration is called the visual persistence; it was longest (up to $400 \mathrm{msec}$ ) for shorter durations and decreased nearly to zero for a 1,000 -msec stimulus. In the three lightadapted conditions used, persistence reached values of only $100-200 \mathrm{msec}$ for the shorter target stimuli and again decreased nearly to zero for the 1,000 msec stimulus.

When the target stimuli were followed at various SOAs by visual noise masks in a light-adapted state, the results indicated that the perceived duration of each target stimulus was approximately equal to the SOA (although usually slightly greater than the SOA) except for those target stimuli that produced perceived durations that would have been shorter than the SOA in the absence of a masking stimulus. For these stimuli, the masking stimulus had no effect on perceived duration. Haber and Standing suggested that the visual noise mask therefore provided an upper limit for the perceived duration of the target stimulus. This suggestion has been influential and has been used to support interruptive theories of visual masking. A review of these theoretical developments and their relation to visual physiology has been given elsewhere (Felsten \& Wasserman, 1980).

In order to compare the effectiveness of homogeneous masking stimuli with visual noise masks, Haber and Standing also measured the perceived durations of $30-\mathrm{msec}$ target stimuli followed at various SOAs by homogeneous light masks. However, this work was done in dark adaptation. Their 
results indicated that at SOAs shorter than about $150 \mathrm{msec}$, the perceived durations of the target stimuli were longer than the SOA, and that at SOAs greater than $150 \mathrm{msec}$, the perceived durations were shorter than the SOA. Haber and Standing suggested that these results indicated that the homogeneous masking flash acted to reduce persistence (just as a light-adapting field would) but that the homogeneous mask did not act to limit perceived duration sharply by interrupting processing as a visual noise mask apparently did.

The present psychobiological experiment was designed to investigate whether or not a sensory code (cf. Uttal, 1973) for perceived duration existed in the responses of single photoreceptors and whether such a code could account for the psychophysical data obtained in all of the above-described conditions of adaption and masking. The present experiment was, therefore, a replication of Haber and Standing's investigation, employing target and masking stimuli of the same duration and temporal arrangements used by Haber and Standing. However, it would be quite difficult to mimic their spatial arrangements directly at the single photoreceptor level. Ideally, a study of the photoreceptor contribution to visual masking in this paradigm would take simultaneous recordings from many photoreceptors illuminated by appropriately patterned stimuli. But it is not feasible to insert microelectrodes into every photoreceptor simultaneously. Nevertheless, microelectrodes inserted into single photoreceptors can still be used to study separately the receptor responses to all of the possible stimulus configurations that might fall on any particular photoreceptor in a visual masking experiment. There are only four possible stimulus conditions that may occur: the receptor may or may not be stimulated by the target and also may or may not be stimulated by the aftercoming visual mask. These four stimulus configurations can be separately presented to one particular receptor, and its response to each can be analyzed. In this manner, all of the possible receptor responses to a spatially complex masking stimulus configuration can be separately analyzed. This strategy is the same as that used by Ratliff and Hartline (1959) in their study of Mach bands. They also could not record the responses to the Mach band stimulus from all cells simultaneously. Instead, they recorded from one single cell, and, by moving the stimulus across the retina, they were able to analyze the responses for each possible stimulus location separately and thereby reconstruct the result they would have obtained had they been able to record from all cells simultaneously.

In the present psychobiological experiment, receptors that are not illuminated by either the target or masking stimulus produce no responses and pro- vide no information to the investigator about photoreceptor contributions to masking or about the target to the central nervous system.

Receptors that are illuminated by either the target stimulus or the masking stimulus, but not by both, produce signals that represent primarily the incident stimulus (although neural network interactions may subsequently alter the receptor responses to some extent). Information about the target is obviously contained in the array of receptors stimulated by the target only, but this information would be relatively unaffected (at the receptor level) by the masking stimulus. Therefore, the effect of equiluminous noise masks at the receptor level should tend to be less than or equal to the effect of homogeneous flash masks, because the former would allow some of the target information to evade masking if all other variables were kept constant. If the residual target information by itself were adequate, then noise masks would be expected to have a lesser effect than flash masks. If not, then the effects of the two masks would be more similar. ${ }^{1}$

The critical information about the target is contained in those receptors that are stimulated by both target and mask; this portion of the target information is available only to the degree that the target representation survives the mask, that is, to the degree that the target modifies or increments the response produced by the mask alone.

One basic assumption of the present experiment is that we can understand the receptor contribution to pattern masking by studying the incremental response produced by the target, because that increment contains the information vulnerable to masking in the receptor. This assumption has been tested in four previous studies of receptor contributions to masking (Felsten \& Wasserman, 1978, 1979a, 1979b; Wasserman, Lo, \& Easland, 1976) and has been found to be generally useful. This assumption is further supported by the data from a study of visual masking which combined electrophysiological and behavioral measures in individual subjects (Fehmi, Adkins, \& Lindsley, 1969). The monkeys in that experiment could respond to the target only when the experimenters could see an incremental effect of the target on the electrophysiological response to the combined target and mask. Of course, the information present in the receptor array about the spatial and temporal properties of a complex stimulus configuration is undoubtedly used in more central analyses, and thus there may well be postreceptor contributions to masking (cf. Felsten \& Wasserman, 1980). But, regardless of the extent to which this form of masking can be divided between photoreceptor and central mechanisms, the photoreceptor signals are the first transformations of the stimuli, and it is these signals that are processed at sub- 
sequent neural levels, not the stimuli themselves. The responses from the individual photoreceptors to each of the possible stimulus configurations represent the complete inventory of inputs available to more central processors whose function cannot be completely understood without knowledge of the receptor contribution.

Central processing probably involves computations of the differences between the responses in the various receptors in order to extract a more refined representation of the target stimulus information. The existence of such comparisons is the second assumption in the present experiment, and it is not unreasonable: Lateral inhibition, which appears to be a virtually universal property of visual systems, is essentially a computation of the differences in the responses in the receptor array.

\section{METHODS}

Intracellular microelectrode recordings were taken from single photoreceptors in the lateral eye of the Limulus, using techniques that are quite conventional and that have been more fully described elsewhere (Felsten \& Wasserman, 1978, 1979a). Several stimulus presentation arrangements were used: (1) Target stimuli (flashes of light), with durations of $10,50,100,250$, or $500 \mathrm{msec}$ and an intensity of $12 \mathrm{~W} / \mathrm{m}^{2}$, were individually presented to darkadapted photoreceptors. (2) The same stimuli were presented individually to light-adapted photoreceptors. In this latter condition, the receptors were light adapted by being stimulated repeatedly at $10-\mathrm{sec}$ intervals by a $500-\mathrm{msec} 380-\mathrm{W} / \mathrm{m}^{2}$ flash of light. The state of the receptor was monitored by observing the responses to these adapting stimuli. Target stimuli were presented by substituting a single target stimulus for one flash in the series of adapting flashes. After each such substitution, the cell was permitted to return to the light-adapted sensitivity level as regulated by the continuing series of adapting flashes. (3) The same target stimuli were presented to light-adapted receptors followed at SOAs of $50,100,200,300$, and $500 \mathrm{msec}$ by a $500-\mathrm{msec} 380-\mathrm{W} / \mathrm{m}^{2}$ masking flash. In this condition, the adaptation state was again maintained by the repetitive adapting flashes, but these also sometimes served as the masking stimuli. When the target and masking stimuli were presented together, the target flash was simply added to one of the train of masking flashes at an appropriate SOA. After each such trial, the cell was permitted to return to its original sensitivity state as regulated by the continuing series of adapting (masking) stimuli. (4) Under dark-adapted conditions, 30-msec target flashes were presented, followed at SOAs of 10,50,100,150,200,300, and $500 \mathrm{msec}$ by a 500 -msec masking flash.

\section{Response Measures}

If a sensory code exists for perceived duration at the receptor level, a response measure will exist that accounts for the human psychophysical estimates of perceived duration in all of the above conditions. When a single stimulus was presented to a darkor light-adapted receptor without an aftercoming masking stimulus, a sensory code was sought that was equal in duration to the perceived duration produced by an equivalent stimulus in a human psychophysical experiment.

When the target stimulus was followed by a masking stimulus, the neural representation of the target stimulus that survived masking and was therefore available for central neural processing, was taken as the incremental response produced by the target stimulus in the presence of the masking stimulus. The incremental response was determined by subtracting the response to the masking flash delivered alone from the response to the combined presentation of target and masking flashes, using a PDP-12 computer and a program (EEG Data Collection, BNI Series, Decus No. 12-1) designed to record and analyze analog neural signals. A sensory code was sought in the incremental response that was equal in duration to the perceived duration of the masked target stimulus in the psychophysical investigation. If the neural correlate of the perceived duration turned out to be the same in both the unmasked and masked conditions, one would have confidence that a true sensory code was being uncovered. As will be seen below, a code that satisfied these constraints turned out to be the time that the receptor response (or the response increment) was above a criterion potential.

The photoreceptor potential in Limulus generates spikes in the eccentric cell that propagate to the brain along the optic nerve fibers, which are the axons of the eccentric cells. The frequency of the spike potentials in the optic nerve fiber is proportional to the amplitude of the receptor potential above a threshold potential (Fuortes, 1958). Therefore, the recoding of visual information from a graded receptor potential to a spike frequency code causes the loss of receptor information that is below the threshold for spike generation. This also produces a spike-train representation of the stimulus that is briefer than the receptorpotential representation of the same stimulus. This might be taken to suggest that a candidate neural code for the perceived duration of a stimulus might be the duration of the spike-train response generated at the pacemaker, for this would correspond to the time that the receptor response was above spike threshold. Despite the inherent attractiveness of this simple candidate code, one should a priori accept the notion that a particular physiological threshold (in this case, the potential required to elicit an action potential) mediates a behavior that also is at threshold. There is no reason to presuppose that the two thresholds are the same, even though we use the same term in both cases. Indeed, Wasserman $(1978,1981)$ found that a Limulus receptor response far greater in magnitude than that required for the threshold generation of spikes in the eccentric cell was required to produce a threshold behavioral response in Limulus. Moreover, Easland and Wasserman (1979) showed that the spike threshold itself varies with light adaptation. Therefore, in our attempt to find a sensory code for perceived duration in the photoreceptor response, we considered a number of possibilities, not just the threshold for spike generation, and we compared the results given by these alternatives with the psychophysical estimates of perceived duration obtained under the same stimulus conditions.

\section{RESULTS}

Recordings were taken from seven retinula (photoreceptor) cells. In six of these investigations, the cells were stimulated by the variable-duration target stimuli alone and also by the same target stimuli followed at various SOAs by the masking stimuli, all under light-adapted conditions. In three of these six cells, the target stimuli were also presented alone in the dark-adapted state. In one of these six cells and in one additional cell, 30 -msec target stimuli were presented, followed at various SOAs by the masking stimuli under dark-adapted conditions.

Figure 1 shows the responses to five target stimuli presented to a photoreceptor without aftercoming masking flashes under dark-adapted and light-adapted conditions. The horizontal dashed lines represent a criterion potential of $11 \mathrm{mV}$. The suggested neural analogs of perceived duration would be as shown in 


\section{dark adapted} light adapted

\section{Test Stimulus Duration (msec)} 500


250
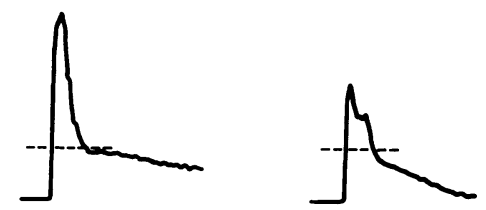

100
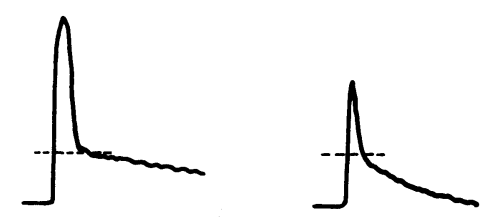

50
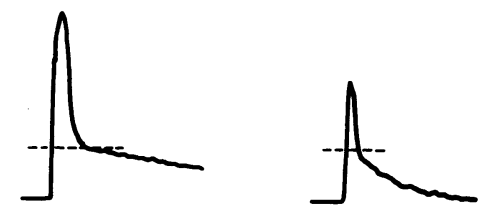

10

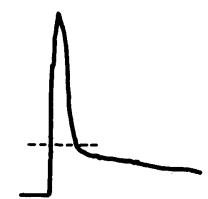

that it might not have been unreasonable to have used a lower criterion for the dark-adapted data than for the light-adapted data. But this would simply have increased the numerical values obtained in that condition without changing the form of the results in any interesting way.

When the target stimuli were followed by masking flashes, we measured the duration of the incremental response that remained above a criterion potential. Figure 3 shows the way that this analysis was performed, using data from another photoreceptor kept in a light-adapted state. A 100 -msec (left column) or a 500-msec (right column) target stimulus was followed at an SOA of 300 msec by a 400 -msec masking stimulus. (In this one experiment, a $400-\mathrm{msec}$ masking flash was used instead of the 500 -msec flash used in the other experiments.) Reading from top to bottom in this figure, the successive panels represent the response to the test flash alone, the response to the masking flash alone, the response to both test and masking flashes given together, and the incremental response to the test flash given in the presence of the masking flash. Using a 14-mV criterion potential (which is indicated by the horizontal dashed lines), the durations of the receptor responses above the criterion potential (or the receptor analogs of perceived duration for the 100 - and 500 -msec target stimuli) are 157 and $539 \mathrm{msec}$, respectively, when unmasked stimuli are used (top row). But the durations of the receptor analogs of perceived duration-namely, the incremental responses-for the masked 100- and 500 -msec stimuli are 154 and $284 \mathrm{msec}$, respectively (bottom row). Thus, at this SOA, the mask truncates

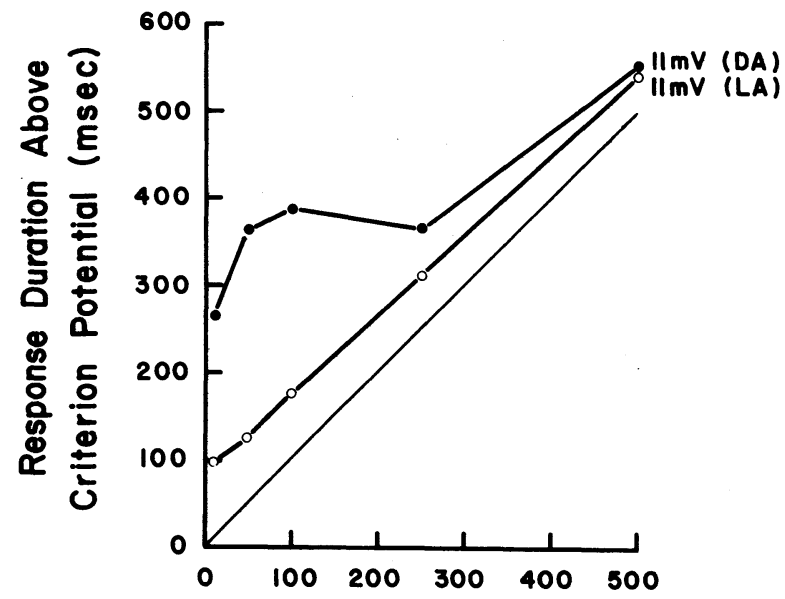

Test Stimulus Duration (msec)

Figure 2. The duration of the receptor response to a single stimulus that remains above a criterion potential of $11 \mathrm{mV}$ as a function of stimulus duration in dark- (DA) and light-adapted (LA) conditions. Data taken from the responses shown in

Figure 1.
Figure 1. Receptor responses to target stimuli presented without aftercoming masking stimuli under dark- and light-adapted contons. Responses to target stimuli of 10, 50, 100, 250, and $\mathbf{5 0 0}$ msec durations are shown. Gain is indicated by the horizontal dashed line, which represents a criterion potential amplitude of $11 \mathrm{mV}$. Time scale is given by the response durations that remain above this criterion potential as plotted in Figure 2. As an example, the analog of perceived duration for the dark-adapted response to the $\mathbf{5 0 0}$-msec flash is $\mathbf{5 5 2} \mathrm{msec}$. (These traces were thickened by hand for reproduction.)

Figure 2 if, for this particular task, the portions of the responses at potentials greater than $11 \mathrm{mV}$ carried information, while information contained in portions of the responses below $11 \mathrm{mV}$ was lost. These psychobiological estimates of perceived duration are qualitatively and quantitatively very similar to those obtained from Haber and Standing's human psychophysical experiments. Similar results were obtained in the other two cells investigated. Varying the criterion potential chosen varied the results, but a criterion potential that approximated the psychophysical results could always be found. Evidence to be given below suggests 

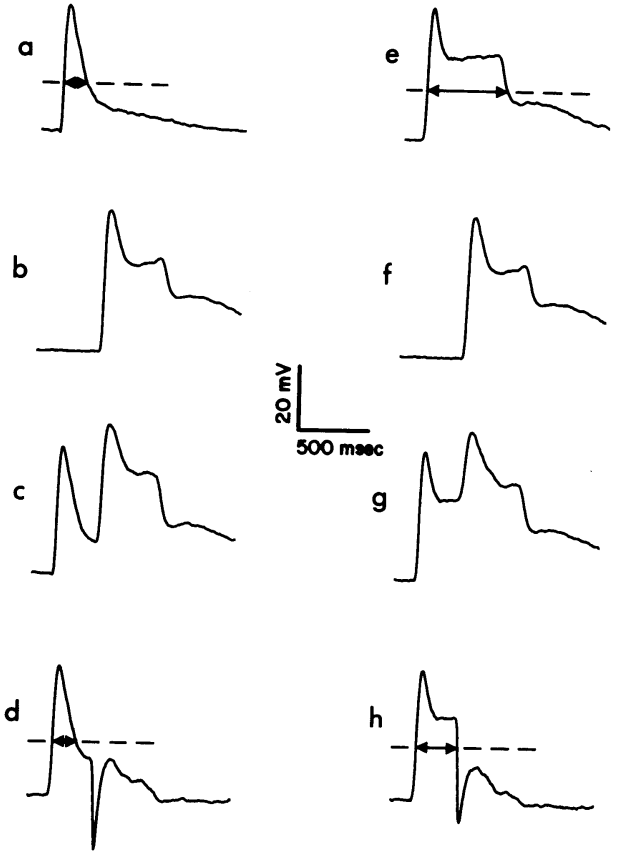

Figure 3. Light-adapted receptor responses to masked and unmasked 100- and 500-msec target stimuli. Panels a and e show the responses to the 100 - and 500 -msec target stimuli presented alone, respectively. Panels $b$ and $f$ are the responses to the masking stimuli presented alone. Panels $\mathrm{c}$ and $\mathrm{g}$ are the responses to the 100 - and $500-$ msec target stimuli presented together, respectively, with the masking stimuli at an SOA of 300 msec. Panels $d$ and $h$ are the incremental responses to the target stimuli obtained by subtracting the responses to the masking stimuli from the responses to the combined presentation of masking stimuli and target stimuli. The horizontal dashed lines represent a criterion potential of $14 \mathrm{mV}$. Response durations at this potential (indicated by arrows) are: (a) $157 \mathrm{msec}$, (d) 154 msec, (e) 539 msec, and (h) $284 \mathrm{msec}$.

the perceived duration of a long response but hardly affects the perceived duration of a short one.

The results from a complete masking experiment in one cell are shown in Figure 4, in which the duration of the incremental response to the target stimulus that remains above a criterion potential is plotted as a function of target stimulus duration, with SOA as a parameter. The SOAs are emphasized by the dashed horizontal lines. In this light-adapted state, it is apparent that the SOA provides an approximate upper limit to the duration of the receptor analog of perceived duration, except for those stimuli that produced receptor analogs of perceived duration that were shorter than the SOA when the target was presented alone. The thin line connecting the stars in Figure 4 relates the duration of the receptor analog of perceived duration to the target stimulus duration for those target stimuli that were presented without any aftercoming masking stimulus. This single-flash function provides the upper limit to the duration of the neural analogs of perceived duration whenever perceived duration is shorter than the SOA.
Using the same general analysis with other criterion potentials resulted in different values of the response analog of perceived duration, but a $14-\mathrm{mV}$ criterion provided data that were most harmonious with the psychophysical data under both the masked and unmasked conditions. The results in the five other cells used for these three conditions were qualitatively similar: Higher criterion potentials produced shorter neural analogs of perceived duration, and vice versa. Furthermore, for each cell, a criterion potential that produced results that were quite consistent with the psychophysical findings for both masked and unmasked target stimuli could be found. This potential varied from one cell to another, but remained within a range of about 6 to $14 \mathrm{mV}$. This variation will be considered further in the discussion section.

There is a minor difference between our lightadapted results and the light-adapted results of Haber and Standing. In our work, the limiting value of perceived duration is slightly shorter than the SOA, whereas, in their work, the limiting value was slightly longer. This suggests that transmission of the coded information to higher centers is accompanied by a slight increase in temporal dispersion. This is exactly what one would expect from existing knowledge of the passive electrical properties of neural membranes, and direct demonstrations of this dispersive effect exist (cf. Kong \& Wasserman, 1978).

We also investigated the duration of the neural ana$\log$ of perceived duration for 30 -msec target stimuli pre-

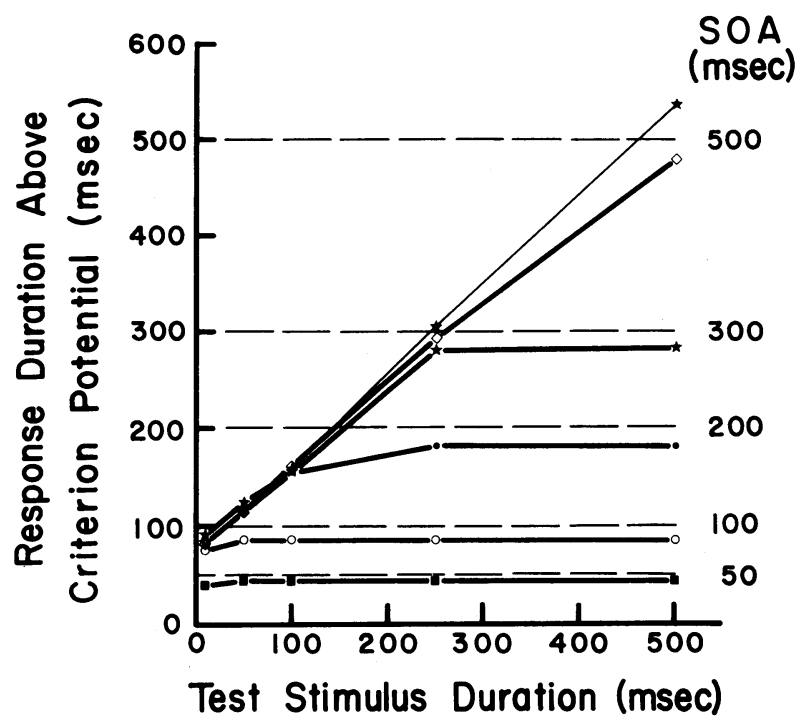

Figure 4. Duration above a $14-\mathrm{mV}$ criterion potential of the incremental responses to target stimuli followed by masking stimuli under light-adapted conditions as a function of actual target stimulus duration with SOA as a parameter. The horizontal dashed lines indicate the SOAs used. The line connecting the stars indicates the durations above the criterion potential of the responses to the same target stimuli presented without aftercoming masking stimuli. 
sented with aftercoming masking stimuli at various SOAs in dark-adapted photoreceptors. This experiment is analogous to Haber and Standing's "blanking flash" experiment. Figure 5 shows how this analysis was performed. As was the case in Figure 3 above, the dashed lines in Figure 5 indicate the time that either the receptor potential or the potential increment is above a criterion. The left column of Figure 5 shows the results obtained with a 50-msec SOA; the right column shows results with a $500-\mathrm{msec}$ SOA. At the top are the target responses alone. At the bottom are the incremental responses to the target when followed by an aftercoming masking flash. Dark adaptation changes the character of the responses. This is perhaps most noticeable in Panel g of Figure 5, where the initial transient of the response to the masking flash is virtually absent. Thus, the earlier test flash alters the response to the later masking flash in this state of adaptation.

The mask truncates the perceived duration of the target at the short SOA but not at the long SOA. But in contrast with the results obtained in the lightadapted conditions (shown in Figures 3 and 4), the


Figure 5. Dark-adapted receptor responses to masked and unmasked 30-msec target stimuli. Panels a and e show the responses to the target stimuli alone. Panels $b$ and $f$ show the responses to the masking stimuli alone. Panels $c$ and $g$ are the responses to the combination of target and mask with 50- and 500-msec SOAs, respectively. Panels $d$ and $h$ are the incremental responses to the target stimuli obtained by subtracting the responses to the masking stimuli from the responses to the combined presentation of masking and target stimuli. The horizontal dashed lines represent a criterion potential of $4.8 \mathrm{mV}$.

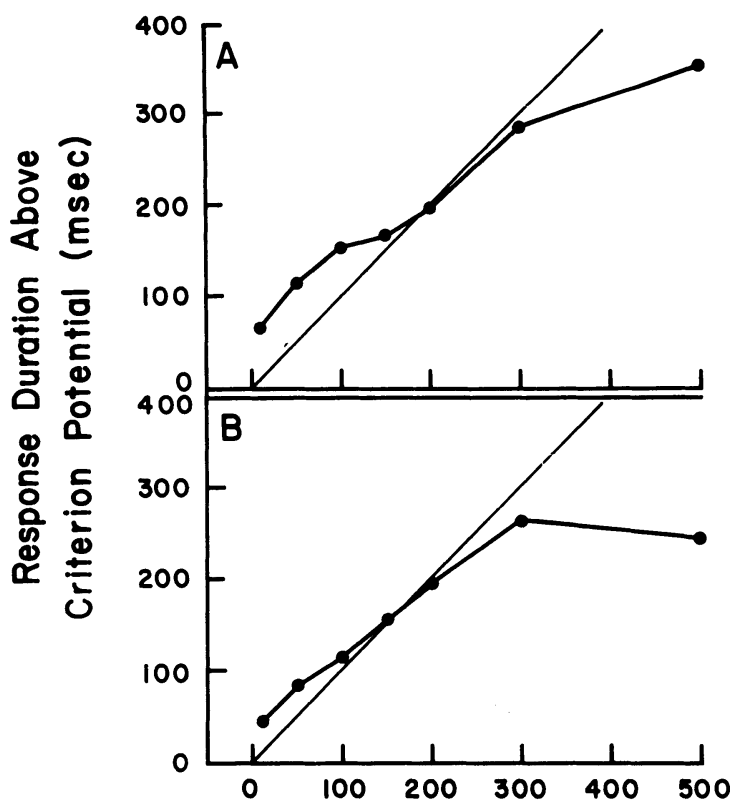

Stimulus Onset Asynchrony (msec)

Figure 6. Duration of the receptor responses above a criterion potential as a function of SOA for 30-msec target stimuli followed at various SOAs by homogeneous masking stimuli under darkadapted conditions. The criterion potential for the data in Panel $A$ was $4.5 \mathrm{mV}$, while that used to obtain the data in Panel B was $4.8 \mathrm{mV}$. The oblique line in each panel indicates the response durations that equal the SOA. Data are shown for two different cells because dark-adapted responses are more variable than light-adapted responses.

truncation leaves a dark-adapted neural analog of perceived duration that is related to the SOA in a more complex way. Figure 6 shows these results quantitatively in two cells. Figure $6 \mathrm{~A}$ shows the duration of the response to the $30-\mathrm{msec}$ flash that remained above a criterion potential of $4.5 \mathrm{mV}$ as a function of SOA in one cell; Figure 6B shows the same analysis for a different cell using a criterion potential of $4.8 \mathrm{mV}$. The results from two cells are given here because dark-adapted visual responses are more variable than light-adapted responses, as is well known. These results are qualitatively and quantitatively quite similar to those reported in dark adaptation by Haber and Standing. At short SOAs, the perceived duration is somewhat longer than the SOA; at long SOAs, the perceived duration is somewhat shorter than the SOA. The criterion potentials chosen here are smaller than those used to obtain the light-adapted data presented above; it should be noted that spike threshold is lower in dark adaptation (Easland \& Wasserman, 1979), and hence one might expect the effective criterion to decrease with dark adaptation even if everything else remained constant.

\section{DISCUSSION}

The results indicate that the duration (above an ap- 
propriate criterion potential) of the receptor response to a target stimulus exhibits the same functional characteristics as the perceived duration of a similar target stimulus presented to a human observer in a psychophysical experiment. This parallelism exists for target stimuli that are presented either alone or with aftercoming masking stimuli, and it holds in both light-adapted and dark-adapted conditions. The results also indicate that the most critical variable in Haber and Standing's experiment was the state of adaptation rather than the spatial structure of their stimuli. Psychophysical data on the effect of spatial structure on perceived duration when adaptation is held constant are not now available, to our knowledge. The present data indicate that, at the receptor level, a visual noise mask should have an effect on limiting perceived duration that is less than or equal to the effect of a homogeneous flash mask. Finding that a noise mask had a greater effect on perceived duration than an equiluminous flash mask would implicate a postreceptor contribution (see Footnote 1).

The most appropriate criterion potential is that which can account for the perceived duration of the target stimulus under all conditions of masking and adaptation. The data indicate that the duration of the proposed neural analog of perceived duration varied as a function of the criterion potential used, but that for each cell investigated, a unique criterion potential could be found that could account parsimoniously for the psychophysical findings under all conditions investigated. Variation in the value of the criterion potential that best accounted for the psychophysical data would be expected because electrotonic conduction of the receptor potential in the retinula cell is decremental. Since the location of the electrode in relation to the site of origin of the potential cannot be kept constant from one cell to another, the amplitude of the potential actually recorded varies as well.

Because this investigation compares neural responses from one species with behavioral data from another species, the numerical values of the criterion potentials found in our physiological investigation may not be the same as those that might be found in any other species-most importantly, in man. However, the general form of the vertebrate photoreceptor response is similar in most respects to that of Limulus (and of most other species, in fact), despite the difference in response polarity. We suggest that the same feature of the neural response-namely, the duration of the receptor response above a criterion potential-is probably the sensory code for perceived duration in human receptors. It is extremely unlikely that the relation between the duration of the proposed neural analog of perceived duration and perceived duration measured psychophysically would otherwise be fortuitously maintained over such a wide variety of conditions. Indeed, the phyletic distance between
Limulus and man suggests that we are dealing with a universal psychobiologic effect (like lateral inhibition) rather than an idiosyncratic species-specific phenomenon. Convergent evidence in favor of this interpretation has been given by Hawkins and Shulman (1979) in their analysis of the effect of luminance on perceived duration, as reported by human subjects. They harmonized the psychophysical literature on this problem by suggesting that instructions can lead a subject to assess either the time when the subject's sensation changes by a criterion amount (Type 1 persistence) or when the subject's sensation exceeds a criterion level (Type II persistence). As defined by Hawkins and Shulman, Type II persistence is equivalent to the sensory code reported here.

The determination of a sensory code for perceived duration complements previous determinations of the sensory codes for stimulus detection (Felsten \& Wasserman, 1978; Wasserman, Felsten, \& Easland, 1979) and stimulus identification (Felsten \& Wasserman, 1979b; Wasserman et al., 1979) and supports the generalization drawn from this work (Wasserman \& Kong, 1979).

Another implication of these data also exists: Haber and Standing (1970) suggested that their results implied "a process-stopping interpretation of information processing by visual noise, as distinct from a sensory integration view" (p. 216). The primary support for this interruption model for masking was the finding that perceived duration was almost exactly defined by the interval between the onset of the target stimulus and the onset of the masking stimulus (the stimulus onset asynchrony). In the absence of direct psychobiological evidence and in the context of the oversimplified view of neural integration that prevailed at the time, Haber and Standing's suggestion was not at all unreasonable.

But the results of the present psychobiological experiments lend strong support to integration theories of visual masking and show how such theories can accommodate the time-dependent data that generated interruptive theories by appealing to time-dependent integrative effects in receptor cells. Panels $\mathrm{c}$ and $\mathrm{g}$ in Figures 3 and 5 show that the sensory signals to the target and masking stimuli are indeed integrated within the most peripheral sensory element, the photoreceptor. This integration is very nonlinear, producing a sensory signal that is quite different from the algebraic sum of the responses to the target and masking stimuli presented separately. Panels $\mathrm{d}$ and $\mathrm{h}$ in Figures 3 and 5 show the effects of this integration on the representation of the target stimuli in a masking paradigm. These incremental responses differ considerably from the responses to the same stimuli presented alone, as given in Panels a and e. The major difference is a rather abrupt termination of the incremental response, characterized by a rapid fall toward baseline and a greatly reduced response amplitude for 
portions of the response occurring at times approximately equal to the SOA (in the light-adapted case). Thus, the duration of the incremental response that remains above a criterion potential (our sensory code for perceived duration) is almost exactly equal to the SOA (in the light-adapted case) because of a timeand energy-dependent integrative interaction between the responses to the target and masking stimuli, which results in an incomplete and degraded representation of the target stimulus in the photoreceptor signal. In the dark-adapted case, a time-dependent influence of the test flash response on the masking flash response complicates the data, but, again, receptor integration produces results that compare well with behavior.

Since it appears that it is the interaction between target and masking stimuli that causes the perceived duration of the target stimulus to be governed by the SOA and that this interaction actually occurs in the photoreceptor, it is no longer necessary to postulate a central interruptive mechanism that causes a cessation in the processing or extraction of information from a complete target stimulus representation. Thus, these data support a sensory integration model of visual masking and not a central interruption model. To date, no physiological support for such an interruption model has been reported, and, indeed, it is difficult to imagine a neural substrate for such a model.

It has been postulated that visual persistence in a photoreceptor might be the icon (Adelson, 1978; Sakitt, 1975). The present interpretation would lead one to say that a masking stimulus interferes with the icon through an integrative mechanism, resulting in a degraded and shortened neural representation of the target stimulus, and not through a process that terminates information extraction from a complete icon.

Although the neural mechanism subserving visual masking appears to be response integration, one could readily infer that a backward mask interrupts the central processing of the target stimulus representation because a peripheral integrative mechanism interrupts the information to be processed. But this usage of the term interruption would have an implication very different from that of its normal usage, which implies a central nonintegrative interaction. One would have to say that the extraction of information from the target stimulus representation cannot be completed because that representation is interrupted, not because processing stops.

The peripheral interaction between the responses to the target and masking stimuli is clearly an energyand time-dependent integration within the photoreceptor. This integration must occur regardless of the particular masking paradigm used, as long as both target and masking stimuli illuminate the same receptors. The mechanisms that mediate masking when the target and masking stimuli do not illuminate the same receptors (i.e., in dichoptic masking and in metacontrast) probably occur at more central neural loci, and further investigations of these more central processes are warranted. One would expect to find central integrative mechanisms that would produce functional relations quite different from those that occur in the receptor, since, in central masking, the sensory signals would be interacting after the nonlinear receptor transformation rather than before.

These conclusions have certain implications for the widespread use of backward masking in perceptual and cognitive psychology. Backward masks are usually assumed to limit the amount of time available to extract information from the representation of a target stimulus. The data presented here suggest that this does not actually occur. Instead, backward masking results in an incomplete and truncated representation of the target stimulus.

However, there is an additional interpretation of these data: When a target stimulus is presented alone, the experimenter has little control over the persistence of the representation of the target stimulus. A 1-msec stimulus may easily produce a sensory response that goes on for hundreds of milliseconds. But by using backward masking, the investigator can rather precisely control the duration of the representation of the target stimulus. Thus, when a backward mask is used in a cognitive experiment, the length of processing time is not under experimental control. Instead, the length of time that the information is available for processing is under experimental control because backward masking interrupts sensory signals by integration.

We suggest that it might be profitable to reanalyze the data from experiments in which masking stimuli were used to control the amount of time available for processing, in terms of the sensory signal available for processing rather than in terms of the extent of processing.

\section{REFERENCES}

Adelson, E. H. Iconic storage: The role of rods. Science, 1978, 201, 544-546.

Easland, G. S., \& Wasserman, G. S. Multiple intracellular contributions to light adaptation in Limulus ommatidia. Vision Research, 1979, 19, 1-8.

Fenmi, L. G., Adkins, J. W., \& Lindsify, D. B. Electrophysiological correlates of visual perceptual masking in monkeys. Experimental Brain Research, 1969, 7, 299-316.

Felsten, G., \& Wasserman, G. S. Masking by light in Limulus receptors. Journal of Comparative and Physiological Psychology, 1978, 92, 778-784.

Felsten, G., \& Wasserman, G. S. Masking-induced sensitivity changes in Limulus photoreceptors. Vision Research, 1979, 19, 943-945. (a) 
Felsten, G., \& Wasserman, G. S. The photoreceptor sensory code for pattern identification during visual masking. Sensory Processes, 1979, 3, 230-279. (b)

Felsten, G., \& Wasserman, G. S. Visual masking: Mechanisms and theories. Psychological Bulletin, 1980, 88, 329-353.

Fuortes, M. G. F. Electrical activity of cells in the eye of Limulus. American Journal of Ophthalmology, 1958, 46, 210-233.

Haber, R. N., \& Standing, L. Direct estimates of the apparent duration of a flash. Canadian Journal of Psychology, 1970, 24, 216-229.

Hawkins, H. L., \& Shulman, G. L. Two definitions of persistence in visual perception. Perception \& Psychophysics, 1979, $25,348-350$.

Kong, K. L., \& Wasserman, G. S. Temporal summation in the receptor potential of the Limulus lateral eye: Comparison between retinula and eccentric cells. Sensory Processes, 1978, 2, 9-20.

Ratliff, F., \& Hartline, H. K. The response of Limulus optic nerve fibers to patterns of illumination on the receptor mosaic. Journal of General Physiology, 1959, 42, 1241-1255.

SAкітт, B. Locus of short term visual storage. Science, 1975, 190, 1318-1319.

Schultz, D. W., \& Eriksen, C. W. Do noise masks terminate target processing? Memory \& Cognition, 1977, 5, 90-96.

Sperling, G. Successive approximations to a model for short term memory. Acta Psychologica, 1967, 27, 285-292.

UTTAL, W. R. The psychobiology of sensory coding. New York: Harper \& Row, 1973.

WASSERman, G. S. Limulus psychophysics: Temporal summation in the ventral eye. Journal of Experimental Psychology: General, 1978, 107, 276-286.

WASSERman, G. S. Limulus psychophysics: Increment threshold. Perception \& Psychophysics, 1981, 29, 251-260.

Wasserman, G. S., Felsten, G., \& Easland, G. S. The psychophysical function: Harmonizing Fechner and Stevens. Science, 1979, 204, 85-87.

Wasserman, G. S., \& Kong, K. L. Absolute timing of mental activity. Behavioral and Brain Sciences, 1979, 2, 243-254.

Wasserman, G. S., Lo, J., \& Easland, G. S. A modified multiplicative rule describes backward masking in a photoreceptor. Vision Research, 1976, 16, 785-788.

\section{NOTE}

1. "Equiluminous" here means that each dot in a noise mask has the same luminance as a homogeneous flash mask (when luminance is related to the number of photons per unit area).
It does not mean that the total number of photons coming from the two masks is equal. The latter condition would produce results that could not be predicted unless one knew precisely the location of each dot in the noise mask relative to the target. A specific example will clarify this point: Consider the task of identifying a target that is either an " $E$ " or an " $F$ " subtending $.4 \mathrm{deg}$ vertically and $.2 \mathrm{deg}$ horizontally. Consider a noise mask that is a single .1-deg-diam dot placed either in register with the lower horizontal stroke of the "E" or anywhere else. Consider, also, a homogeneous flash mask subtending $10.0 \mathrm{deg}$ centered on the target. Now take up the case of equal luminance of the noise dot and the homogeneous flash. If the dot is in register with the lower stroke of the "E," then the receptor effect of the noise dot mask will be equal to that of the flash mask. Alternatively, if the dot is anywhere else, the crucial target information will evade noise masking at the receptor level and the noise mask will be less effective than the flash mask.

However, if the noise dot mask contains the same number of photons as does the entire homogeneous flash mask, then the luminance of the noise dot mask will be $4 \mathrm{log}$ units greater than that of the flash dot mask. As a consequence, the noise dot mask will be far more effective than the flash mask when the dot is in register with the critical target information. But when the dot is not in register, the noise dot mask will produce no receptor masking.

Thus, the equal luminance case generates a clear-cut prediction-the noise mask should always yield a lesser or equivalent degree of receptor masking. The equal photon case does not yield any prediction-the noise mask will sometimes be less effective and sometimes be more effective-unless one knows the registration exactly. This simple example can be generalized to more typical masking situations in two steps. First, let the noise dot have a random location from trial to trial and let the structure of the critical target information be arbitrary. No element of these predictions will change. Second, let there be many randomly located noise dots. This would produce a quantitative change; the 4-log-unit factor mentioned above would decline in proportion to the number of dots. However, the direction of all predictions would remain the same and registration information would still be needed to predict the results when the photon number is kept constant. Finally, the ability of the registration variable to produce opposite results from noise masks when photon number is held constant has been directly demonstrated by Schultz and Eriksen (1977).

(Received for publication July 27, 1980; revision accepted October 7,1980 .) 